\title{
COMPARACIÓN DEL RENDIMIENTO Y CAMBIOS MORFOLÓGICOS EN MAÍZ TUXPEÑO V-520C ADAPTADO A VALLES ALTOS EN MÉXICO
}

\author{
COMPARISON OF YIELD AND MORPHOLOGICAL CHANGES IN V-520C \\ TUXPEÑO MAIZE ADAPTED TO HIGHLANDS IN MEXICO
}

\section{Fernando López-Morales ${ }^{1}$, J. Jesús García-Zavala **, Tarsicio Corona-Torres ${ }^{1}$, Serafín Cruz-Izquierdo', Gustavo López-Romero², Delfino Reyes-López³ Ma. Gricelda Vásquez-Carrillo ${ }^{4}$ y José D. Molina-Galán ${ }^{1+}$}

\begin{abstract}
${ }^{1}$ Colegio de Postgraduados (CP), Campus Montecillo, Postgrado en Recursos Genéticos y Productividad-Genética, Montecillo, Texcoco, Estado de México, México. ${ }^{2} \mathrm{CP}$, Campus Veracruz, Postgrado de Agroecosistemas Tropicales, Tepetates, Manlio Fabio Altamirano, Veracruz, México. ${ }^{3}$ Benemérita Universidad Autónoma de Puebla, Facultad de Ingeniería Agrohidráulica, San Juan Acateno, Teziutlán, Puebla, México. ${ }^{4}$ Instituto Nacional de Investigaciones Forestales, Agrícolas y Pecuarias, Campo Experimental Valle de México, Coatlinchán, Texcoco, Estado de México, México.
\end{abstract}

*Autor de correspondencia (zavala@colpos.mx)

\section{RESUMEN}

La selección masal visual (SMV) es un método efectivo para adaptar razas tropicales y subtropicales de maíz (Zea mays L.) a otros ambientes. La variedad de maíz Tuxpeño V-520C es originaria de tierras bajas de Veracruz, pero fue adaptada por SMV a Valles Altos durante 14 (C14) y 19 (C19) ciclos, seleccionando plantas sanas y con buen rendimiento. El objetivo de este estudio fue evaluar el rendimiento de grano y cambios morfológicos de planta y mazorca en la variedad original V-520C (C0), C14 y C19, junto con el Compuesto Universal (CU) de la raza Chalqueño como testigo. En los ciclos agrícolas de 2013 y 2014 se realizaron seis experimentos en los estados de México y Veracruz. El diseño experimental fue bloques completos al azar con tres repeticiones. Se registraron el rendimiento de grano, características agronómicas, de espiga y componentes del rendimiento. Los análisis de varianza combinado a través de localidades e individual de Veracruz no incluyeron el CU, mientras que el de Valles Altos sí lo hizo. Se observaron cambios morfológicos significativos por efecto de la SMV en el análisis combinado y en el de Valles Altos, y en los tres análisis hubo al menos una significancia entre ambientes, entre genotipos o en la interacción genotipo por ambiente para rendimiento y caracteres morfológicos de planta y mazorca. $\mathrm{El}$ C19 incrementó las frecuencias de genes favorables para mayor rendimiento en el análisis combinado $\left(3.5 \mathrm{t} \mathrm{ha}^{-1}\right)$ y de Veracruz $\left(2.6 \mathrm{t} \mathrm{ha}^{-1}\right)$; además, redujo los días a floración, la altura de planta y el tamaño de espiga, e incrementó el número de ramas primarias de la espiga y los componentes de rendimiento; debido a ésto, C19 fue el mejor genotipo, similar al CU. La SMV fue efectiva para la adaptación del maíz Tuxpeño V-520C de la zona tropical a la zona templada.

Palabras clave: Zea mays, adaptación, caracteres agronómicos, caracteres de espiga, componentes de rendimiento, raza Tuxpeño, selección masal.

\section{SUMMARY}

Visual mass selection (VMS) is an effective method to adapt tropical and subtropical races of maize (Zea mays L.) to other environments. The Tuxpeño V-520C maize variety is native to the lowlands of Veracruz, but was adapted to highlands through VMS for 14 (C14) and 19 (C19) cycles by selecting healthy and good yielding plants. This study evaluated grain yield and morphological changes in plant and ear at the original variety $\mathrm{V}-520 \mathrm{C}$ (C0), C14 and C19, along with Compuesto Universal (CU) of the Chalqueño race as a control. In the 2013 and 2014 agricultural cycles, six experiments were carried out in the states of Mexico and Veracruz. A randomized complete blocks design with three replications was used. Grain yield, agronomic traits, tassel characteristics and yield components were recorded. The combined analysis of variance across localities and the individual one for Veracruz did not include CU while analysis for the highlands did. There were significant morphological changes due to VSM in the combined and highland analyses. There was significance between environments, between genotypes or in the genotype-by-environment interaction for grain yield and morphological traits of plant and ear in all the three analyses. C19 increased favorable gene frequencies for higher yield in the combined analysis $\left(3.5 \mathrm{t} \mathrm{ha}^{-1}\right)$ and in that of Veracruz (2.6 tha-1); also, it reduced days to flowering, plant height and tassel size, and increased the number of primary branches of the tassel and yield components. Because of this, $\mathrm{C} 19$ was the best genotype, similar to $\mathrm{CU}$. Visual mass selection was effective for adaptation of Tuxpeño V-520C maize from the tropical zone to the temperate zone.

Index words: Zea mays, adaptation, agronomic traits, mass selection, tassel characteristics, Tuxpeño race, yield components.

\section{INTRODUCCIÓN}

El maíz (Zea mays L.) es el cereal cultivado más importante del mundo, pues sobrepasa en producción al trigo (Triticum aestivum L.) y arroz (Oryza sativa L.), y únicamente es superado por la producción de caña de azúcar (Saccharum officinarum L.) en tallo (FAOSTAT, 2019). El maíz es el componente básico de la alimentación humana y animal, y también es la materia prima para la industria agropecuaria en México y otros países, como Estados Unidos de América, China, Argentina y Brasil (OCDE-FAO, 2014).

México se considera el centro de origen, domesticación y diversificación del maíz, por lo que el territorio nacional cuenta con una gran diversidad genética y entre su población existe gran cantidad de usos y costumbres para su consumo alimenticio (Kato et al., 2009), pero su 
rendimiento promedio nacional fue de apenas de $3.8 \mathrm{t}$ ha${ }^{1}$ de grano para el año 2018 (SIAP, 2019), el cual es bajo comparado con el de los principales países productores; por ejemplo, para el mismo año, el rendimiento promedio en los Estados Unidos de América fue de $10.1 \mathrm{t} \mathrm{ha}^{-1}$ (USDA, 2019).

López-Morales et al. $(2017 ;$ 2019) señalaron que las razas de maíz de México son poco utilizadas en los programas de mejoramiento genético por no contar con adaptación y estabilidad, por lo que estos programas están limitados a pocas razas locales: Tuxpeño en el trópico húmedo, Celaya y Cónico Norteño en el Bajío, y Chalqueño y Cónico en los Valles Altos. Al respecto, existen evidencias de germoplasma de maíz tropical y subtropical adaptado a climas templados mediante selección masal visual (SMV) (Pérez et al., 2002; Pérez-Colmenarez et al., 2007); específicamente, la raza Tuxpeño se pudo adaptar a Valles Altos de México (> 2200 msnm) con este método (Pérez-Colmenarez et al., 2000).

La raza Tuxpeño de maíz se distingue por la calidad de su grano, buen vigor de planta y mazorca, cierta tolerancia a la sequía y resistencia a plagas y enfermedades (Bellón et al., 2005; Wen et al., 2012), por lo que es muy usada en cruzamientos de materiales mejorados por poseer alto rendimiento y amplia capacidad de adaptación en muchas otras zonas del país (López-Morales et al., 2017; 2019). Esta raza predomina en el estado de Veracruz, en altitudes de 0 a 500 m, donde prevalece el clima cálido húmedo (López-Morales et al., 2014).

El germoplasma de maíz exótico tropical y subtropical puede aportar nuevos alelos útiles al material local, principalmente para resistencias a plagas, enfermedades y estreses ambientales como la sequía (Vazquez-Pozos et al., 2020), y puede aumentar la variabilidad genotípica y con ello ampliar la base genética para mejorar las poblaciones localmente adaptadas a las zonas templadas como los Valles Altos; tal germoplasma exótico puede ser usado también de manera per se como nuevas variedades alternas y complementarias al maíz local y contribuir a la diversidad del maíz en la zona agrícola templada (Goodman y Carson, 2000).

No obstante, las razas tropicales y subtropicales de maíz no se adaptan fácilmente a ambientes templados, a menos que se cuente con una estrategia adecuada. La SMV es una opción para este fin, ya que, a través de varios ciclos de selección, las variedades exóticas presentan características similares o superiores a los materiales locales, lo que podría generar un incremento en la diversidad genética del maíz local (Pérez et al., 2002; Pérez-Colmenarez et al., 2000; 2007), o el material adaptado podría usarse directamente.
La variedad $\mathrm{V}$-520C fue formada originalmente en el Campo Experimental Cotaxtla del Instituto Nacional de Investigaciones Forestales, Agrícolas y Pecuarias con germoplasma nativo de la raza Tuxpeño, fue sometida a 19 ciclos de SMV para su adaptación a Valles Altos de México con base en el criterio de seleccionar plantas rendidoras y sanas. El objetivo de este estudio fue evaluar el rendimiento y los cambios morfológicos de planta y mazorca de la variedad V-520C raza Tuxpeño adaptada mediante SMV en Valles Altos. Se postula que la SMV aplicada en las plantas de maíz sanas y rendidoras aumentan la frecuencia de genes favorables para rendimiento y adaptación, y que tal selección, causa cambios morfológicos en planta y mazorca en los genotipos más aptos y de mayor avance de selección; por lo tanto, la SMV será efectiva para adaptar el maíz Tuxpeño de zona tropical a zona templada.

\section{MATERIALES Y MÉTODOS}

\section{Material genético}

La variedad de maíz V-520C ciclo cero (C0) de la raza Tuxpeño se sometió a 19 ciclos de selección masal visual (SMV) en el Valle de México durante 19 años consecutivos (1989-2008), un ciclo por año, obteniéndose semilla de los compuestos de los ciclos de selección C14 y C19. La selección se hizo visualmente considerando aspectos de la mazorca (larga, cilíndrica, sana, con hileras rectas y grano blanco dentado) y de la planta (sana, vigorosa, sin acame, típica de Tuxpeño). La SMV de los ciclos 14 y 19 se llevó a cabo en el Campo Agrícola Experimental del Colegio de Postgraduados, Campus Montecillo, ubicado en Texcoco, con clima templado subhúmedo (García, 2004) y una altitud de $2250 \mathrm{~m}$. En el experimento se incluyó como testigo a la variedad de polinización libre compuesto universal SM12 (CU) de la raza local Chalqueño para comprobar la similitud del material seleccionado con el local.

La SMV empezó aproximadamente con 510 plantas sanas en cada ciclo, haciendo cruzamientos fraternales entre las plantas de mayor sanidad y fenotipo. Inicialmente se seleccionaban todas las mazorcas, pero después, cuando ya se producía más material, se seleccionó de manera visual sólo las mejores 50 mazorcas para formar el compuesto de cada ciclo de selección.

\section{Ubicación de los experimentos}

La V-520C de los ciclos cero (C0), C14, C19 y el testigo CU se evaluaron en los años 2013 y 2014 en Montecillo y en 2014 en Coatlinchán, municipio de Texcoco, Estado de México. Una tercera localidad se estableció en el predio Tepetates del municipio de Manlio Fabio Altamirano, Veracruz, con tres experimentos en 2013 y 2014, por lo 
que en total se tuvieron seis ambientes (Cuadro 1). El diseño experimental empleado fue bloques completos al azar con tres repeticiones, donde la unidad experimental consistió de dos surcos de $6 \mathrm{~m}$ de longitud, separados a $80 \mathrm{~cm}$, con dos semillas sembradas cada $50 \mathrm{~cm}$, para un total de 52 plantas por parcela, equivalente a una densidad de población de 50 mil plantas por hectárea.

\section{Manejo agronómico}

La fórmula de fertilización empleada en los dos ciclos de evaluación en Montecillo (M 2013-PV y M 2014-PV) fue 140N-60P-00K, mientras que en Coatlinchán (C 2014PV) se utilizó la fórmula 140N-40P-00K (Cuadro 1). Al momento de la siembra se aplicó todo el fósforo y la mitad del nitrógeno; la otra mitad de nitrógeno se aplicó a los 35 días después de la siembra. En las dos localidades se aplicó un riego inicial de germinación y se dieron riegos de auxilio cuando fue necesario. En los tres ciclos de Tepetates ( $T$ 2013-PV, T 2014-Ol y T 2014-PV) se fertilizó con la fórmula $110 \mathrm{~N}-46 \mathrm{P}-00 \mathrm{~K}$, se aplicó 64N-18P-00K al momento de la siembra y el resto a los 30 días después de la siembra. En el predio de Tepetates se aplicó riego cada 10 días sólo al ensayo establecido en el ciclo OI, desde la siembra hasta el llenado de grano, mientras que los ensayos establecidos en los ciclos PV en los años 2013 y 2014 fueron de secano. Para el control de maleza en todos los casos, se aplicó herbicida con los ingredientes activos dicamba, atrazina, terbutrina, paraquat y nicosulfurón, con las dosis que se recomiendan de cada producto para el cultivo de maíz en las regiones respectivas.

\section{Variables evaluadas}

El rendimiento (REN) en $t$ ha $^{-1}$ se estimó con el peso de mazorca por parcela, con ajuste de humedad al $14 \%$. También, en cada parcela experimental se registraron los promedios de 10 plantas con competencia completa o de 10 mazorcas, para caracteres agronómicos y de espiga, y componentes del rendimiento, respectivamente (LinaresHolguín et al., 2019; López-Romero et al., 2005; RocandioRodríguez et al., 2014). Los caracteres agronómicos evaluados fueron días a floración masculina (DFM) y femenina (DFF), como el número de días transcurridos desde la siembra hasta que el $50 \%$ de las plantas de la parcela presentaron antesis y estigmas expuestos en el jilote superior, respectivamente; también se midieron la altura de planta (APL) y la altura de inserción de la mazorca superior (AMZ) en cm. En la espiga se midió la longitud total (LTE), longitud del segmento ramificado (LSR) y longitud de la rama central (LRC) en $\mathrm{cm}$, desde el pedúnculo, inicio de ramificación e inicio de la rama central hasta el ápice de la rama central, respectivamente; así mismo, se contabilizó el número de ramas primarias (NRP). Los componentes del rendimiento medidos en cada mazorca fueron la longitud (LMZ) y diámetro (DMZ) en cm y el número de hileras (NHM).

\section{Análisis estadístico}

Se realizó análisis de varianza combinado con datos de los seis ambientes de evaluación (Cuadro 1) sin considerar a la variedad CU, ya que este material no se desarrolló de

Cuadro 1. Características edafoclimáticas de las seis localidades de evaluación de los maíces Tuxpeños: $\mathrm{C} 0, \mathrm{C} 14, \mathrm{C} 19, \mathrm{y}$ CU de los estados de México y Veracruz.

\begin{tabular}{|c|c|c|c|c|c|c|c|}
\hline \multirow{2}{*}{$\begin{array}{l}\text { Localidad, } \\
\text { año-ciclo }^{+}\end{array}$} & \multirow{2}{*}{$\begin{array}{l}\text { Abreviatura } \\
\text { localidad }\end{array}$} & \multirow{2}{*}{ Altitud (m) } & \multirow{2}{*}{$\begin{array}{c}\text { Fórmula de } \\
\text { fertilización } \\
\text { N-P-K }\end{array}$} & \multirow{2}{*}{$\begin{array}{c}\text { Tipo de } \\
\text { suelo, pH }\end{array}$} & \multirow{2}{*}{$\begin{array}{l}\text { Precipitación }{ }^{+\dagger} \\
\quad(\mathrm{mm})\end{array}$} & \multicolumn{2}{|c|}{$\mathrm{T}\left({ }^{\circ} \mathrm{C}\right)^{x x}$} \\
\hline & & & & & & Máx & Mín \\
\hline $\begin{array}{l}\text { Montecillo, } \\
\text { 2013-PV }\end{array}$ & M 2013-PV & 2250 & $140-60-00$ & Vertisol, 7.5 & $1277^{\bullet}$ & 29.0 & 6.0 \\
\hline $\begin{array}{l}\text { Montecillo, } \\
2014-P V\end{array}$ & M 2014-PV & 2250 & $140-60-00$ & Vertisol, 7.5 & $1179^{\circ}$ & 29.0 & 3.0 \\
\hline $\begin{array}{l}\text { Coatlinchán, } \\
\text { 2014-PV }\end{array}$ & C 2014-PV & 2300 & $140-40-00$ & Phaeozem, 6.2 & $1306^{\bullet 9}$ & 30.0 & 6.0 \\
\hline $\begin{array}{l}\text { Tepetates, } \\
\text { 2013-PV }\end{array}$ & T 2013-PV & 20 & $110-46-00$ & Vertisol, 6.3 & 1227 & 35.9 & 21.2 \\
\hline $\begin{array}{l}\text { Tepetates, } \\
2014-01\end{array}$ & T 2014-OI & 20 & $110-46-00$ & Vertisol, 6.3 & $26^{x}$ & 42.2 & 10.6 \\
\hline $\begin{array}{l}\text { Tepetates, } \\
\text { 2014-PV }\end{array}$ & T 2014-PV & 20 & $110-46-00$ & Vertisol, 6.3 & 1975 & 34.8 & 20.2 \\
\hline
\end{tabular}

${ }^{\dagger}$ Año de siembra en ciclos OI (otoño-invierno) y PV (primavera-verano), ${ }^{\dagger \dagger}$ Precipitación total acumulada del periodo siembra-cosecha, ${ }^{\natural}$ Riego inicial + tres riegos auxiliares, “Riego inicial, ${ }^{x}$ Diez riegos auxiliares, respectivamente; ${ }^{x \times T e m p e r a t u r a s ~ M a ́ x ~(m a ́ x i m a) ~ y ~ M i ́ n ~(m i ́ n i m a) ~ d u r a n t e ~ e l ~ p e r i o d o ~}$ siembra-cosecha. 
manera satisfactoria en las tres evaluaciones de Tepetates, Veracruz, por estar desadaptado a las condiciones de clima cálido subhúmedo. Para el análisis de varianza de los tres ciclos agrícolas en las localidades de Valles Altos sí se incluyó al CU. En un tercer análisis de varianza para Veracruz sólo se incluyeron los tres ciclos agrícolas de evaluación y se analizaron los datos de los tres materiales Tuxpeños. Los análisis de varianza y la comparación de medias por la prueba de Tukey $(P \leq 0.05)$ se realizaron con el programa estadístico SAS® versión 9.0 (SAS Institute, 2002).

\section{RESULTADOS Y DISCUSIÓN}

En los resultados del análisis de varianza combinado y de Veracruz, sin el CU (Cuadro 2), la significancia fue similar entre las fuentes de variación ambientes y genotipos para las variables de rendimiento (REN), días a floración masculina y femenina (DFM y DFF), altura de planta y de mazorca (APL y AMZ), características de espiga en general y para todos los componentes del rendimiento, excepto número de hileras por mazorca (NHM). En contraparte, la interacción genotipo por ambiente (IG $\times A$ ) en Veracruz fue significativa sólo para cuatro de las 12 variables, mientras que en el análisis combinado nueve variables presentaron significancia en la IG $\times$ A. La similitud entre el análisis combinado y de Veracruz para REN se debió a que ambos análisis contaron con genotipos de maíz Tuxpeño, lo que se confirma con los cuadrados medios de ambientes para esta variable (11.56 y 11.01) y sus medias fenotípicas (2.48 y $2.15 \mathrm{t} \mathrm{ha}^{-1}$ ); en cambio, las diferencias entre estos dos análisis fueron los efectos de los cuadrados medios sobre los genotipos y la IG × A (Cuadro 2).

En el análisis de varianza de Valles Altos (Estado de México) se detectó significancia entre ambientes para ocho de las 12 variables, excepto REN, LRC, NRP y NHM, lo que indica que la expresión promedio de estas cuatro variables fue muy similar a través de los ambientes de Valles Altos. Entre genotipos hubo significancia para todas las variables, debido a que, además de los materiales Tuxpeños, se tuvo a la variedad local compuesto universal $(\mathrm{CU})$, lo cual provocó que en este análisis se tuvieran los cuadrados medios más altos entre genotipos y en la IG $\times$ A para casi todas las variables, esto en comparación con los dos análisis que sólo tuvieron materiales Tuxpeños.

El coeficiente de variación (CV) de los tres análisis resultó alto para REN (34.8 a $40.6 \%$ ), mientras que el resto de las variables tuvo valores aceptables (Cuadro 2). Las significancias de los análisis de varianza de Veracruz y del Estado de México indican el contraste entre los ambientes y la diferente expresión genética de los genotipos. Los resultados coinciden con lo encontrado por López-Morales et al. (2017; 2019), ya que se utilizaron los compuestos de selección de la variedad Tuxpeño V-520C evaluados tanto en Valles Altos como Veracruz, donde la SMV produjo cambios significativos entre los ciclos C0, 14 y 19 para rendimiento y características de calidad de grano y tortilla; así mismo, los resultados se ajustan a lo encontrado por López-Morales et al. (2014), Rocandio-Rodríguez et al. (2014) y Linares-Holguín et al. (2019) en trabajos similares para datos morfológicos de planta y mazorca.

\section{Cambios en el rendimiento}

Hubo significancia $(P \leq 0.01)$ para REN entre ambientes, entre genotipos y en la $I G \times A$ en el análisis combinado (Cuadro 2). Estos resultados se debieron a que las condiciones ambientales de las dos zonas geográficas son diferentes en altitud, precipitación, tipo de suelo, $\mathrm{pH}$ y temperaturas, tal como se observa en el Cuadro 1; además, entre los genotipos las diferencias se debieron al rendimiento contrastante entre ciclos de SMV; por lo tanto, la combinación de efectos genéticos de genotipos y efectos ambientales ocasionó significancia en la IG $\times$ A.

La comparación de medias (Tukey, $\mathrm{P} \leq 0.05$ ) entre genotipos (Cuadro 3) indica que en el análisis combinado el C19 avanzado tuvo el rendimiento promedio más alto, 3.54 t ha-1 ${ }^{-1}$ lo que superó a la media de Valles Altos de $3.0 \mathrm{t} \mathrm{ha}^{-1}$ (SIAP, 2019), seguido por el C14 y C0, con un rendimiento promedio de 2.29 y $1.62 \mathrm{t} \mathrm{ha}^{-1}$, respectivamente. Los resultados del promedio de REN indican que la SMV fue efectiva para mejorar el rendimiento de la variedad Tuxpeño V-520C, ya que el C19 superó a sus ciclos anteriores, teniendo la población una respuesta positiva a la selección.

El análisis de varianza para Valles Altos detectó significancia entre genotipos y en la IG $\times$ A para REN (Cuadro 2). Entre ambientes no hubo significancia, lo que pudo deberse a que se tuvieron siembras en dos ciclos agrícolas de la localidad de Montecillo (M 2013-PV y M 2014-PV), y otra en la localidad de Coatlinchán (C 2014PV), la cual es muy cercana a la de Montecillo. La variedad local CU de Chalqueño tuvo el mejor rendimiento con 6.79 t ha- ${ }^{-1}$, seguido por el C19 de Tuxpeño con 4.43 t ha $^{-1}$, el C14 y CO con promedio de 2.55 y $1.46 \mathrm{t} \mathrm{ha}^{-1}$, respectivamente (Cuadro 3). La prueba de medias indicó que el CU fue superior en REN al C19 hasta en $35 \%$, a pesar de haber realizado la SMV recurrente en Valles Altos; por lo tanto, el C19 fue más cercano al CU que los ciclos anteriores. La significancia entre genotipos concuerda con lo reportado por Pérez et al. (2002) y Pérez-Colmenarez et al. (2007) para el mismo material Tuxpeño V-520C, y Pérez-Colmenarez et al. (2000) con germoplasma Tuxpeño Crema 1, en ambas investigaciones se evaluó el rendimiento mejorado por SMV sólo en Valles Altos de México. 


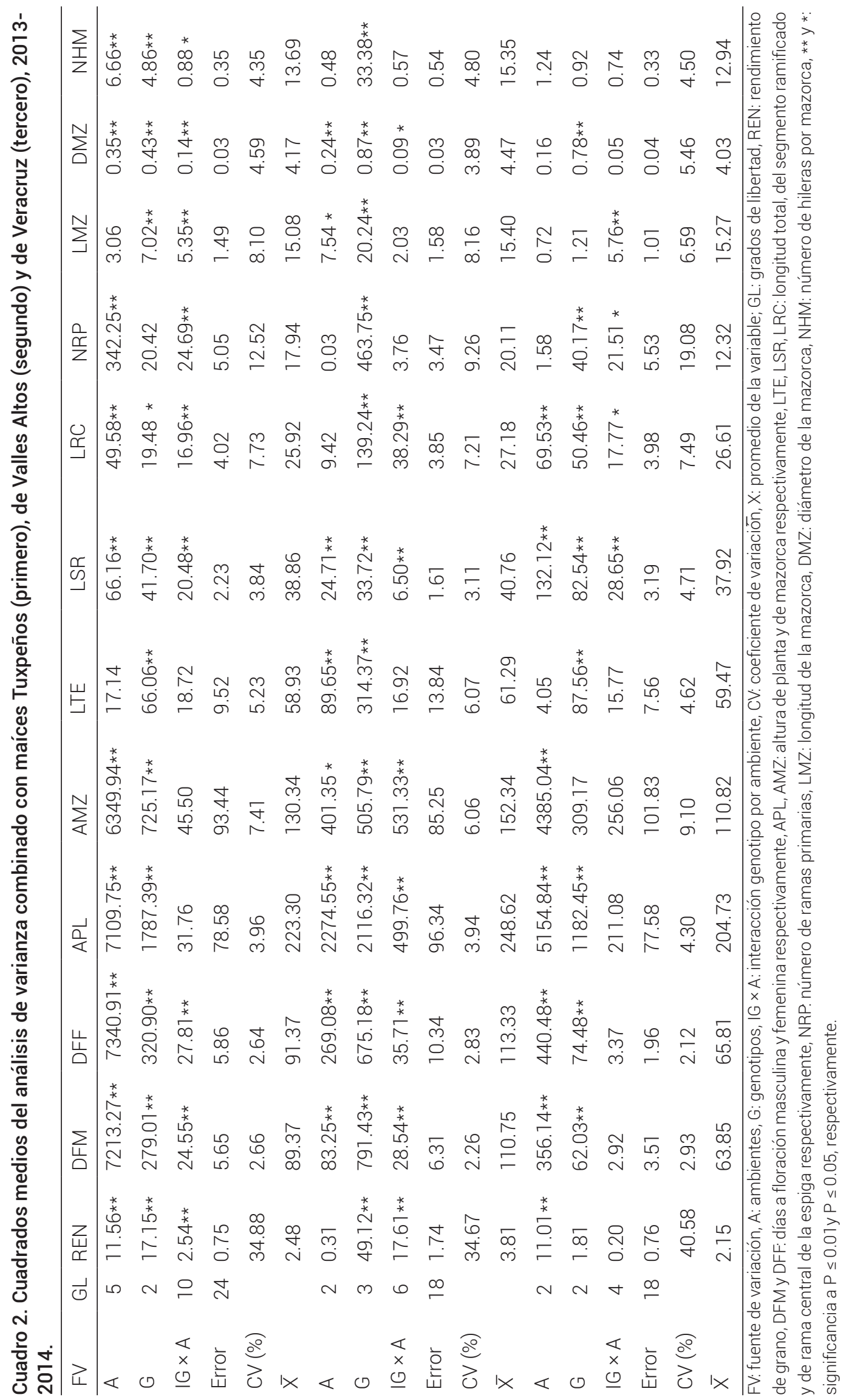


Cuadro 3. Promedios de 12 variables del análisis combinado con maíces Tuxpeños (primero), promedios de Valles Altos (segundo) y promedio del estado de Veracruz (tercero), 2013-2014.

\begin{tabular}{|c|c|c|c|c|c|c|c|c|c|c|c|c|}
\hline Ciclo & $\begin{array}{c}\text { REN } \\
\left(\mathrm{t} \mathrm{ha}^{-1}\right)\end{array}$ & $\begin{array}{c}\text { DFM } \\
\text { (días) }\end{array}$ & $\begin{array}{c}\text { DFF } \\
\text { (días) }\end{array}$ & $\begin{array}{l}\mathrm{APL} \\
(\mathrm{cm})\end{array}$ & $\begin{array}{l}\text { AMZ } \\
(\mathrm{cm})\end{array}$ & $\begin{array}{l}\text { LTE } \\
(\mathrm{cm})\end{array}$ & $\begin{array}{l}\text { LSR } \\
(\mathrm{cm})\end{array}$ & $\begin{array}{l}\text { LRC } \\
(\mathrm{cm})\end{array}$ & NRP & $\begin{array}{l}\mathrm{LMZ} \\
(\mathrm{cm})\end{array}$ & $\begin{array}{l}\mathrm{DMZ} \\
(\mathrm{cm})\end{array}$ & NHM \\
\hline $\mathrm{CO}$ & $1.62 b$ & $91.88 a$ & $94.66 a$ & $233.35 a$ & $136.91 a$ & $59.59 a$ & $38.96 b$ & $25.81 a b$ & $17.35 a$ & $14.38 b$ & $4.24 a$ & $3.26 b$ \\
\hline C14 & $2.29 b$ & $91.38 a$ & $92.83 a$ & $223.14 b$ & $129.85 a b$ & $60.42 a$ & $40.32 a$ & $27.01 a$ & $17.31 a$ & $15.58 a$ & $3.99 b$ & $13.53 b$ \\
\hline C19 & $3.54 a$ & $84.83 b$ & $86.61 b$ & $213.42 \mathrm{c}$ & $124.25 b$ & $56.77 b$ & $37.28 \mathrm{c}$ & $24.93 b$ & $19.17 a$ & 15.28ab & $4.28 a$ & $14.27 a$ \\
\hline DSH & 0.72 & 1.98 & 2.01 & 7.37 & 8.04 & 2.56 & 1.24 & 1.66 & 1.87 & 1.01 & 0.15 & 0.49 \\
\hline $\mathrm{CO}$ & $1.46 c$ & 119.00a & $122.44 a$ & 249.38b & $157.15 a$ & $57.52 b$ & $39.93 b$ & $25.34 b$ & $20.82 b$ & 13.34b & $4.21 \mathrm{c}$ & $13.93 c$ \\
\hline C14 & $2.55 c$ & 116.88a & 117.66b & $244.31 b c$ & $148.86 a b$ & $60.01 b$ & $39.74 b$ & $24.88 b$ & $24.70 a$ & $15.78 a$ & $4.29 b c$ & $14.07 \mathrm{c}$ \\
\hline C19 & $4.43 b$ & $108.77 b$ & $110.66 c$ & $231.95 c$ & $143.54 b$ & $57.64 b$ & $39.72 b$ & $25.44 b$ & $25.18 a$ & $15.54 a$ & $4.45 b$ & $15.31 b$ \\
\hline $\mathrm{CU}$ & $6.79 a$ & $98.33 c$ & $102.55 d$ & $268.83 a$ & $159.82 a$ & $69.98 a$ & $43.66 a$ & $33.07 a$ & $9.75 c$ & 16.93a & $4.91 a$ & $18.08 a$ \\
\hline DSH & 1.76 & 3.34 & 4.28 & 13.07 & 12.30 & 4.95 & 1.69 & 2.61 & 2.48 & 1.67 & 0.23 & 0.98 \\
\hline $\mathrm{CO}$ & $1.77 a$ & $64.77 a$ & $66.88 a$ & $217.32 \mathrm{a}$ & $116.67 a$ & $61.66 a$ & $38.00 \mathrm{~b}$ & $26.27 b$ & $13.88 a$ & $15.42 a$ & $4.27 a$ & $12.60 a$ \\
\hline C14 & $2.02 a$ & $65.88 a$ & $68.00 a$ & $201.97 b$ & $110.84 a$ & $60.84 a$ & $40.91 a$ & $29.13 a$ & $9.92 b$ & $15.37 a$ & $3.70 b$ & $13.00 \mathrm{a}$ \\
\hline C19 & $2.64 a$ & $60.88 \mathrm{~b}$ & $62.55 b$ & 194.90b & $104.95 a$ & $55.90 \mathrm{~b}$ & $34.85 \mathrm{c}$ & $24.43 b$ & $13.16 a$ & $15.02 a$ & $4.12 a$ & $13.23 a$ \\
\hline DSH & 1.09 & 3.51 & 1.76 & 11.07 & 12.69 & 3.45 & 2.24 & 2.51 & 2.95 & 1.26 & 0.27 & 0.33 \\
\hline
\end{tabular}

C0, C14, C19: Ciclos 0, 14 y 19, respectivamente de selección masal visual de V-520C raza Tuxpeño; CU: compuesto universal de la raza Chalqueño; DSH: diferencia significativa honesta; REN: rendimiento de grano; DFM, DFF: días a floración masculina y femenina, respectivamente; APL, AMZ: altura de planta y de mazorca, respectivamente; LTE, LSR, LRC: longitud total, del segmento ramificado y de rama central de la espiga, respectivamente; NRP. número de ramas primarias; LMZ: longitud de la mazorca; DMZ: diámetro de la mazorca; NHM: número de hileras por mazorca. Medias con distinta letra en las columnas dentro de cada análisis son estadísticamente diferentes (Tukey, $\mathrm{P} \leq 0.05$ ).

Por otro lado, el análisis de varianza de los tres ensayos en la localidad de Tepetates en el estado de Veracruz (Cuadro 2) detectó significancia entre ambientes para REN, donde sólo se incluyeron los tres genotipos de la raza Tuxpeño (Cuadro 3). La significancia entre ambientes pudo deberse a que dos ensayos se evaluaron en el periodo de primavera-verano y uno en otoño-invierno; es decir, las condiciones variaron en los tres ambientes de prueba (Cuadro 1).

\section{Cambios adaptativos en caracteres agronómicos}

De acuerdo con los análisis de varianza combinado y de Valles Altos, hubo significancia entre ambientes, entre genotipos y en la IG $\times$ A para DFM y DFF, mientras que en el análisis de varianza de Veracruz sólo hubo significancia entre ambientes y entre genotipos para las dos variables (Cuadro 2). La significancia de la IG $\times$ A entre el análisis de Veracruz y el análisis combinado fue contrastante porque en Veracruz los ensayos se llevaron a cabo en la misma localidad, lo cual redujo el contraste ambiental. En el análisis combinado los genotipos C0 y C14 tuvieron medias similares para las dos variables y diferentes con respecto a C19, que fue el más precoz.

La prueba de medias de Valles Altos indicó que los genotipos C0 y C14 tuvieron los mayores promedios; para
DFM fueron de 119 y $116.8 \mathrm{~d}$, respectivamente, mientras que el C19 tuvo $108.7 \mathrm{~d}$ y el material local CU tuvo el promedio más bajo con $98.3 \mathrm{~d}$. Para DFF, el C0 tuvo 122.4 d, seguido por C14 con 117.6 d, C19 con 110.6 d y CU con $102.5 \mathrm{~d}$ (Cuadro 3); esto indica que el CU fue el material más precoz de esta zona geográfica para las dos variables, por ser una variedad originaria de Valles Altos, localmente adaptada, seguido por el genotipo C19 que disminuyó sus características de precocidad acercándose en ambas variables al $\mathrm{CU}$, lo que indica su afinidad al material original.

Lo recomendable para adaptar la raza Tuxpeño a Valles Altos es disminuir el número de días a la floración, ya que así se evitaría el riesgo por problemas con heladas tempranas y se aumentaría el periodo de llenado de grano (Ángeles-Gaspar et al., 2010); por lo tanto, el mejor genotipo con estas características fue el $\mathrm{CU}$, que tuvo 10.4 DFM y 8.1 DFF menos con respecto al C19; no obstante, el C19 fue 10.3 DFM y 11.8 DFF más precoz con respecto al Co; en este contexto, se ha documentado que la SMV recurrente es un método eficaz para reducir los DFM y DFF, seleccionando plantas precoces. Así, Pérez-Colmenarez et al. (2000; 2007) y Pérez et al. (2002) lograron reducir los DFM en 17.1, 3.2 y 5.6 d, respectivamente, con relación al CO, en la variedad Tuxpeño Crema 1 (el primero) y Tuxpeño V-520C (los dos últimos), seleccionados bajo el método de SMV y evaluados en clima templado. 
En la prueba de medias aplicada a los experimentos de Veracruz los genotipos C0 y C14 tuvieron valores similares altos en DFM (64.7 y 65.8, respectivamente) y DFF (66.8 y 68.0, respectivamente), mientras que el C19 tuvo promedios de 60.8 y 62.5 , respectivamente, para cada variable, lo cual indica que se avanzó en precocidad con respecto a los ciclos inferiores, tal como se comprobó en la zona de Valles Altos para las dos variables.

La altura de planta (APL) y la altura de la mazorca superior (AMZ) tuvieron significancia $(P \leq 0.01)$ para ambientes y genotipos en el análisis combinado, mientras que en el análisis de varianza de Valles Altos, además de ambientes y genotipos, también la IG $\times$ A resultó significativa para las dos variables; en cambio, en Veracruz APL tuvo significancia para ambientes y genotipos, mientras que AMZ sólo tuvo significancia para ambientes (Cuadro 2).

En el análisis combinado, la prueba de Tukey detectó diferencias significativas entre genotipos $(P \leq 0.05)$ para APL y AMZ (Cuadro 3). Para APL y AMZ el CO tuvo las plantas más altas; el $\mathrm{C} 14$ fue intermedio, y los promedios más bajos los tuvo el C19, lo cual agronómicamente es más conveniente.

En tanto, en el análisis de Valles Altos la variedad local CU fue el material de mayor APL y AMZ y le siguieron C0 y C14, el más bajo fue C19, lo cual significa avance hacia plantas bajas en los ciclos de selección (Cuadro 3). Lo anterior indica que la SMV es un método efectivo para reducir altura de planta y de mazorca superior, pues en la selección para la adaptación del maíz Tuxpeño a los Valles Altos se buscó seleccionar plantas rendidoras y de bajo porte. Al respecto, Pérez-Colmenarez et al. (2000) obtuvieron en el ciclo de selección más avanzado valores ligeramente mayores de APL y AMZ con respecto al Co, lo que difiere de los resultados de esta investigación, y sugirieron que la APL y AMZ descendieron conforme avanzaba la SMV, donde el C19 quedó con la menor altura de planta y de mazorca, tal como resultó en el análisis combinado. Al respecto, se puede mencionar que esta discrepancia pudo ocurrir por el genotipo utilizado, ya que el material Tuxpeño V-520C evaluado en este trabajo es uno de los ocho que integraron el Tuxpeño Crema 1 evaluado por Pérez-Colmenarez et al. (2000), y por lo tanto debe tener un comportamiento diferente. También en Veracruz se redujo la APL y la AMZ conforme avanzó la SMV, independientemente de que en ese ambiente la AMZ no tuvo significancia para genotipos (Cuadro 2 y 3 ).

Al igual que para rendimiento, los días a floración y altura de planta y mazorca presentaron un contraste marcado entre de las dos zonas geográficas. También se observó que la IG $\times$ A fue significativa en dos de tres análisis de varianza para tales variables, lo que significa que las condiciones edafoclimáticas de cada región tuvieron efectos distintos sobre los genotipos (Cuadro 1).

\section{Cambios adaptativos en las características de espiga}

El análisis de varianza combinado detectó significancia entre ambientes, entre genotipos y en la IG $\times$ A para las variables longitud del segmento ramificado (LSR) y longitud de rama central (LRC), mientras que la longitud total de la espiga (LTE) fue significativa sólo para genotipos. En Valles Altos no hubo significancia en la IG × A para LTE y entre ambientes para LRC. En Veracruz, al igual que en el análisis combinado, no hubo significancia para ambientes e IG × A en LTE (Cuadro 2).

La prueba de medias del análisis combinado indicó que para las tres variables (LTE, LSR y LRC) el C14 tuvo los mayores valores, el C19 los menores y el C0 tuvo valores intermedios. Por otro lado, los promedios en Valles Altos tuvieron significancia, donde la variedad CU tuvo los valores más elevados en LTE, LSR y LRC, mientras que los genotipos de Tuxpeño (CO, C14 y C19) fueron inferiores estadísticamente al CU (Cuadro 3). La comparación de medias del análisis de Veracruz resultó similar a la del análisis combinado, donde el C14 tuvo los mayores promedios, con excepción de la variable LTE, donde el promedio más alto fue para C0 y C19 tuvo los menores promedios en las tres variables (Cuadro 3).

El análisis combinado para número de ramas primarias de la espiga (NRP) detectó significancia entre ambientes y en la IG × A, pero no entre genotipos. En Valles Altos sólo hubo significancia entre ambientes, y en Veracruz sólo hubo significancia entre genotipos y en la IG $\times$ A (Cuadro 2). La prueba de medias del análisis combinado indicó que el C19 tuvo el mayor NRP (19.1), seguido por C0 y C14 (17.3, en ambos) (Cuadro 3). La prueba de Tukey $(P \leq 0.05)$ en Valles Altos indicó que los promedios más bajos los tuvo el CU con valores de 9.7 para NRP, mientras que los ciclos de Tuxpeño tuvieron valores entre 20.8 (C0) y 25.1 (C19). En Veracruz, el promedio de la variable NRP, de mayor a menor, quedó de la siguiente manera: C0, C19 y C14, con valores de 13.8, 13.1 y 9.9. Con respecto a los valores de esta variable, Pérez-Colmenarez et al. (2000) observaron que el NRP disminuye conforme se avanza en la SMV. Posiblemente, en el trópico húmedo el maíz Tuxpeño presenta mayor transpiración por las altas temperaturas, por lo que presenta espigas más grandes y ramificadas, lo que no ocurre en Valles Altos, donde el ambiente es más frío y seco, por lo que los materiales locales tienen espigas pequeñas y poco ramificadas. El contraste observado en el NRP en los materiales evaluados en Valles Altos, excepto el CU, entre este trabajo y el de Pérez-Colmenarez et al. 
(2000) pudo deberse a los diferentes genotipos utilizados en los dos estudios, ya que tales autores utilizaron Tuxpeño Crema 1, mientras en este trabajo se utilizó Tuxpeño V-520C. Otra diferencia en los resultados del NRP se presentó entre la evaluación en Veracruz y la realizada en el trópico húmedo de Puebla por López-Morales et al. (2014), estos autores encontraron un promedio de 20.4 ramas para Tuxpeño Vera-39, lo cual fue 6.5 unidades más elevado con respecto al C0 (13.8); tal diferencia pudo deberse al tipo de variedades evaluadas y al diferencial de altitud de $315 \mathrm{~m}$ entre ambas regiones.

\section{Componentes del rendimiento}

En el análisis combinado hubo significancia $(P \leq 0.01)$ entre genotipos y en la $I G \times A$ para longitud $(L M Z)$ y diámetro de mazorca (DMZ); entre ambientes sólo DMZ fue significativa. En Valles Altos hubo significancia en las tres fuentes de variación para las variables, excepto en la IG $\times$ A para LMZ. En Veracruz hubo significancia en la IG $\times$ A para LMZ y entre genotipos para DMZ (Cuadro 2).

La comparación de medias de Tukey $(P \leq 0.05)$ en el análisis combinado indicó que el promedio más alto en LMZ lo tuvo el C14 con $15.5 \mathrm{~cm}$ y el más bajo el C0 con $14.3 \mathrm{~cm}$ (Cuadro 3), mientras que para DMZ, C19 tuvo el valor mayor con $4.2 \mathrm{~cm}$ y C14 el más bajo con $3.9 \mathrm{~cm}$. En Valles Altos, el CU tuvo los promedios más altos en LMZ y DMZ, con 16.9 y $4.9 \mathrm{~cm}$, y C0 el más bajo con 14.3 y $4.2 \mathrm{~cm}$, respectivamente, pero sólo en DMZ se avanzaba conforme aumentaba el REN con todos los genotipos evaluados en Valles Altos, por ello el CU fue el de mayor valor. Estos resultados concuerdan con los obtenidos por PérezColmenarez et al. (2000), donde el CU tuvo los valores más altos en DMZ, aunque discreparon con la LMZ que tuvo los valores menores. Los resultados de García et al. (2002), al evaluar Tuxpeño Crema 1 con 12 ciclos de SMV en Valles Altos, también coincidieron con el DMZ de este trabajo y con la LMZ de $15.7 \mathrm{~cm}$ del C14 de Tuxpeño V-520C.

En Veracruz, los genotipos no tuvieron diferencias significativas para $L M Z$, pero sí para $D M Z$, donde los ciclos 0 y 19 tuvieron los valores más altos $(4.2$ y $4.1 \mathrm{~cm}$, respectivamente) y el ciclo 14 el más bajo $(3.7 \mathrm{~cm})$. Los resultados indicaron que el ciclo 0 (el más alto), al estar en su ambiente original superó a los demás genotipos, seguido por el ciclo 19, el cual tiene frecuencias alélicas favorables y se acercó al valor de Co, por lo que tuvo el mejor rendimiento en Veracruz.

En las tres fuentes de variación del análisis combinado hubo significancia para el número de hileras por mazorca (NHM), en tanto en Valles Altos, sólo hubo significancia entre genotipos, mientras que en Veracruz no hubo significancia en ninguna fuente de variación (Cuadro 2).

En la comparación de medias de Tukey $(P \leq 0.05)$ del análisis combinado, el C19 resultó el mejor genotipo para la variable NHM, mientras que el genotipo CO fue el de menor valor (Cuadro 3). En Valles Altos, los mejores genotipos fueron la variedad local CU con 18.0 hileras. La variable NHM aumentó significativamente junto con el rendimiento conforme avanzaron el número de ciclos de SMV (Cuadro 3); lo mismo ocurrió con los resultados de Pérez-Colmenarez et al. (2000) en la misma variable de Tuxpeño Crema 1; el mismo comportamiento ocurrió entre los genotipos en Veracruz para esta variable.

\section{CONCLUSIONES}

El maíz tropical Tuxpeño V-520C experimentó cambios morfológicos en planta y mazorca por efecto de la selección masal visual para adaptarse en Valles Altos. En la combinación de las zonas geográficas evaluadas, los cambios mayores ocurrieron en rendimiento de grano y características agronómicas y cambios menores en caracteres de espiga y componentes de rendimiento. La SMV de V-520C en Valles Altos incrementó el rendimiento y sus componentes conforme se avanzó en los ciclos de selección (C0, C14 y C19); en tanto, los caracteres agronómicos y de espiga (excepto el número de ramas primarias) disminuyeron. Los cambios favorables observados en 19 ciclos de SMV indicaron que el maíz Tuxpeño se está adaptando a las condiciones de Valles Altos, con un rendimiento aceptable, reducción en los días a la floración, altura de planta y tamaño de la espiga y tiende a tener características agronómicas similares a las del material local. La SMV fue efectiva para adaptar el maíz Tuxpeño V-520C, de zona tropical a zona templada.

\section{BIBLIOGRAFÍA}

Ángeles-Gaspar E., E. Ortiz-Torres, P. A. López y G. López-Romero (2010) Caracterización y rendimiento de poblaciones de maíz nativas de Molcaxac, Puebla. Revista Fitotecnia Mexicana 33:287-296.

Bellón M. R., M. Adato, J. Becerril and D. Mindek (2005) Impact of Improved Maize Germplasm on Poverty Alleviation: The Case of TuxpeñoDerived Materials in México. International Maize and Wheat Improvement Center. Mexico, D. F. 58 p.

FAOSTAT, Food and Agriculture Organization Corporate Statistical Database (2019) Crops. Food and Agriculture Organization of the United Nations, Rome. http://www.fao.org/faostat/en/\#data/QC/ visualize (June 2016).

García E. (2004) Modificaciones al Sistema de Clasificación Climática de Köppen (Para Adaptarlo a las Condiciones de la República Mexicana). $5^{a}$ edición. Instituto de Geografía, Universidad Nacional Autónoma de México. México. D. F. 98 p.

García Z. J., J. López R., J. Molina G. y T. Cervantes S. (2002) Selección masal visual estratificada y de familias de medios hermanos en una cruza intervarietal $F_{2}$ de maíz. Revista Fitotecnia Mexicana 25:387-391.

Goodman M. M. and M. L. Carson (2000) Reality vs. myth: corn breeding, exotics, and genetic engineering. In: $55^{\text {th }}$ Annual Corn and Sorghum Research Conference Proceedings. American Seed 
Trade Association. Washington, D. C. pp:149-172.

Kato Y. T. A., C. Mapes S., L. M. Mera O., J. A. Serratos H. y R. A. Bye B. (2009) Origen y Diversificación del Maíz: Una Revisión Analítica. Universidad Nacional Autónoma de México, Comisión Nacional para el Conocimiento y Uso de la Biodiversidad. México, D. F. $116 \mathrm{p}$.

Linares-Holguín 0. 0., M. Rocandio-Rodríguez, A. Santacruz-Varela, J. Á. López-Valenzuela, L. Córdova-Téllez, S. Parra-Terraza, ... y P. SánchezPeña (2019) Caracterización fenotípica y agronómica de maíces (Zea mays ssp. mays L.) nativos de Sinaloa, México. Interciencia 44:421-428

López-Morales F., O. R. Taboada-Gaytán, A. Gil-Muñoz, P. A. López and D. Reyes-López (2014) Morphological diversity of native maize in the humid tropics of Puebla, México. Tropical and Subtropical Agroecosystems 17:19-31.

López-Morales F., M. G. Vázquez-Carrillo, J. D. Molina-Galán, J. J. GarcíaZavala, T. Corona-Torres, S. Cruz-Izquierdo, G. López-Romero, D. Reyes-López y G. Esquivel-Esquivel (2017) Interacción genotipoambiente, estabilidad del rendimiento y calidad de grano en maíz Tuxpeño. Revista Mexicana de Ciencias Agrícolas 8:10351050, https://doi.org/10.29312/remexca.v8i5.106

López-Morales F., M. G. Vázquez-Carrillo, J. J. García-Zavala, G. LópezRomero, D. Reyes-López y J. D. Molina-Galán (2019) Estabilidad y adaptación del rendimiento y calidad de tortilla en maíz Tuxpeño, Valles-Altos. Revista Mexicana de Ciencias Agrícolas 10:1809-1821, https://doi.org/10.29312/remexca.v10i8.1851

López-Romero G., A. Santacruz-Varela, A. Muñoz-Orozco, F. Castillo-González, L. Córdova-Téllez y H. Vaquera-Huerta (2005) Caracterización morfológica de poblaciones nativas de maíz del istmo de Tehuantepec, México. Interciencia 30:284-290.

OCDE-FAO, Organización para la Cooperación y el Desarrollo EconómicosOrganización de las Naciones Unidas para la Alimentación y la Agricultura (2014) OCDE-FAO Perspectivas Agrícolas 20142023. OECD Publishing. Paris. 340 p. https://doi.org/10.1787/ agr_outlook-2014-es

Pérez C. A. A., J. D. Molina G. y A. Martínez G. (2002) Adaptación a clima templado de razas tropicales y subtropicales de maíz de
México por selección masal visual. Rendimiento, altura de planta y precocidad. Revista Fitotecnia Mexicana 25:435-441.

Pérez-Colmenarez A. A., J. D. Molina-Galán y Á. Martínez-Garza (2000) Adaptación a clima templado de una variedad de maíz tropical mediante selección masal visual estratificada. Agrociencia 34:533-542.

Pérez-Colmenarez A. A., J. Molina-Galán, Á. Martínez-Garza, P. García-M. y D. Reyes-López (2007) Selección masal para la adaptación a clima templado de razas tropicales y sub-tropicales de maíz de México. Bioagro 19:133-141.

Rocandio-Rodríguez M., A. Santacruz-Varela, L. Córdova-Téllez, H. LópezSánchez, F. Castillo-González, R. Lobato-Ortiz, ... y R. Ortega-Paczka (2014) Caracterización morfológica y agronómica de siete razas de maíz de los Valles Altos de México. Revista Fitotecnia Mexicana 37:351-361.

SAS Institute (2002) User's Guide of SAS (Statistical Analysis System) SAS Institute Inc. Cary, North Carolina, USA. 550 p.

SIAP, Servicio de Información Agroalimentaria y Pesquera (2019) Anuario estadístico de la producción agrícola. Servicio de Información Agroalimentaria y Pesquera, Secretaría de Agricultura y Desarrollo Rural. Ciudad de México. https://nube.siap.gob.mx/ cierreagricola/ (Febrero 2020)

USDA, United States Department of Agriculture (2019) Statistics for subject. National Statistics for Corn. National Agricultural Statistics Service, United States Department of Agriculture. Washington D. C. https://www.nass.usda.gov/Charts_and_Maps/Field Crops/cornyld.php (May, 2020).

Vázquez-Pozos V., E. Serrano-Flores, S. Cruz-Izquierdo y R. Lobato-Ortiz (2020) QTLs asociados con la tolerancia a sequía en una población de maíz tropical utilizando líneas y cruzas de prueba. Revista Fitotecnia Mexicana 43:101-112, https://doi org/10.35196/rfm.2020.1.101-112

Wen W., J. Franco, V. H. Chávez-Tovar, J. Yan and S. Taba (2012) Genetic characterization of a core set of a tropical maize race Tuxpeño for further use in maize improvement. PLoS ONE 7(3):e32626, https://doi.org/10.1371/journal.pone.0032626 
